\title{
Differential Expression of mGluR2 in the Developing Cerebral Cortex of the Mouse
}

\author{
Pooja S. Venkatadri, Charles C. Lee \\ Department of Comparative Biomedical Sciences, School of Veterinary Medicine, Louisiana State University, \\ Baton Rouge, USA \\ Email: cclee@lsu.edu
}

Received 24 August 2014; revised 9 October 2014; accepted 24 October 2014

Copyright @ 2014 by authors and Scientific Research Publishing Inc.

This work is licensed under the Creative Commons Attribution International License (CC BY). http://creativecommons.org/licenses/by/4.0/

(c) (i) Open Access

\begin{abstract}
Glutamatergic synaptic transmission is an essential component of neural circuits in the central nervous system. Glutamate exerts its effects by binding to various types of glutamate receptors, which are found distributed on neurons throughout the central nervous system. These receptors are broadly classified into two main groups, ionotropic glutamate receptors (iGluRs) and metabotropic glutamate receptors (mGluRs). Unlike iGluRs, the mGluRs are G-protein coupled receptors that exert their effects on postsynaptic membrane conductance indirectly through the downstream modification of ion channels. A subtype of mGluRs, the Group II mGluRs, are particularly interesting since their activation by glutamate results in a hyperpolarizing response. Thus, glutamate can act potentially as an inhibitory neurotransmitter, by binding to postsynaptic Group II mGluRs. Given the potential importance of these receptors in synaptic processing, the development of the central nervous system, and neurological disorders, we sought to characterize the expression of mGluR2 in the developing neocortex of the mouse. Therefore, we examined the distribution of mGluR2 in the developing cerebral cortex. We found a general caudal to rostral gradient in the expression of these receptors, with ventral cortical regions labeled caudally and dorsal regions labeled rostrally. Limbic regions highly expressed mGluR2 throughout the brain, as did sensory and motor cortical areas. Finally, other non-cortical structures, such as the thalamic reticular nucleus, amygdala, and mammillary bodies were found to have significant expression of the receptor. These results suggest that mGluR2 may play important roles in mediating glutamatergic inhibition in these structures and also could have a role in shaping the development of mature neural networks in the forebrain.
\end{abstract}

\section{Keywords}

Metabotropic Glutamate Receptor, Mouse, Cortex, mGluR2, Group II mGluR 


\section{Introduction}

Glutamate and its receptors are found throughout the central nervous system [1]-[4]. In the forebrain, glutamate is primarily responsible for the postsytnaptic excitation of neurons through the activation of ionotropic glutamate receptors (iGluRs). Several types of iGluRs are found postsynaptically on neuronal cell bodies and dendrites, such as AMPA and NMDA receptors [1]. Activation of iGluRs by glutamate results in the ligand-gated opening of ion channels, which in turn results in the depolarization of the neuronal membrane, leading to the generation of excitatory postsynaptic potentials.

In contrast to the iGluRs, activation of metabotropic glutamate receptors (mGluRs) does not directly result in the opening of an ion channel [2] [3]. Instead, binding of glutamate to mGluRs results in the activation of a G-protein coupled biochemical cascade that in turn results in the indirect modification of ion channels. There are three main groups of mGluRs (Groups I, II, and III), with each comprised of several different subtypes [3] [5] [6]. Among these the Group II mGluRs are noteworthy, since their activation by glutamate does not result in the depolarizing excitation of the neuronal membrane, but instead results in a hyperpolarizing inhibition [3] [7] [8]. Thus, glutamate can have potentially different effects on neurons, either excitatory or inhibitory, depending on the type of receptor to which it binds.

In other parts of the central nervous system, Group II mGluRs have typically been found on presynaptic terminals [2] [4] [9]-[11]. However, recent findings have also demonstrated postsynaptic localization of Group II mGluRs in some regions of the brain [4] [12] [13]. In the neocortex, we have found previously that these receptors are present both pre- and post-synaptically in the primary sensory areas of the developing mouse cortex [8] [14]. This is intriguing, since these receptors may have special roles in synaptic processing, the development of the central nervous system, and several neurological disorders [5] [15]-[22]. However, the localization of these receptors to other parts of the developing forebrain in the mouse is less well characterized. Therefore, in this study, we sought to examine the distribution of mGluR2 in the developing brain of the mouse.

\section{Materials and Methods}

\subsection{Histology}

To examine the distribution of the Group II mGluR subtype, mGluR2, in the developing brain, we used C57BL6/J mice (ages postnatal days 10 - 13). The Institutional Animal Care and Use Committee of the Louisiana State University School of Veterinary Medicine approved all of these procedures. Mice were anesthetized with isoflurane anesthesia followed by a lethal dose of sodium pentobarbital. Immediately following cessation of reflex responses, the mice were transcardially perfused with a fixative solution containing 4\% paraformaldehyde (Electron Microscopy Sciences, Hatfield, PA) and 50\% saturated picric acid in $0.01 \mathrm{M}$ phosphate buffered saline (PBS). The perfused animals were left for $1 \mathrm{~h}$ prior to dissection of brains, which were then post-fixed overnight in the $4 \%$ paraformaldehyde $/ 50 \%$ saturated picric acid solution. The following day, the brains were transferred to a 4\% paraformaldehyde/30\% sucrose solution for 2 - 3 days for cryoprotection. The brains were blocked coronally using a clean razor blade at the rostral end of the cerebrum and were then sectioned at $50 \mu \mathrm{m}$ using a cryostat (Leica Microsystems, Buffalo Grove, IL). The sections were collected in 0.01 M PBS and then transferred to 24 well plates for processing. Chemical reagents were obtained from Sigma-Aldrich (St. Louis, MO), unless otherwise noted.

The sections were first treated for $30 \mathrm{~min}$ in $0.5 \% \mathrm{H}_{2} \mathrm{O}_{2}$ in order to remove endogenous peroxidase activity. The sections were then rinsed 4 times in $0.01 \mathrm{M}$ PBS, then placed in a solution containing $0.3 \%$ Triton X-100 in $0.01 \mathrm{M}$ PBS for $1 \mathrm{~h}$ to permeablize the cell membranes. The sections were transferred to $1 \%$ normal mouse serum (Vector Labs, Burlingame, CA) in $0.01 \mathrm{M}$ PBS for $1 \mathrm{~h}$ in order to block non-specific binding of the primary antibody. Sections were then incubated at $4^{\circ} \mathrm{C}$ for $24 \mathrm{~h}$ in a 1:1000 dilution of primary antibody solution for mGluR2 (abcam 15672) (Abcam Inc., Cambridge, MA). The next day, sections were rinsed four times in 0.01M PBS and then incubated for $1 \mathrm{~h}$ at room temperature with a biotinylated goat anti-mouse secondary antibody (Mouse ABC Kit, Vector Labs). The sections were then rinsed four times in 0.01M PBS and incubated for $1 \mathrm{~h}$ with the Vectastain avidin-biotin-peroxidase ABC kit (Mouse ABC Kit, Vector Labs). The sections were rinsed four times in 0.01M PBS, then three times in 0.05 M Tris Buffered Saline (pH 7.4) (TBS), before reacting with diaminobenzadine (DAB) (SigmaFast DAB, Sigma-Aldrich). The reaction was quenched by washing three times in TBS, followed by washing four times in PBS. Sections were finally mounted, cleared, and coverslipped. Finally, adjacent sections were Nissl stained for cytoarchitectural analysis [23]. 


\subsection{Microscopy}

The mGluR2 labeled slides were first examined using a Nanozoomer-XR slide scanner (Hamamatsu Photonics, Bridgewater, NJ). The broad distribution of labeling was then compared relative to adjacent cytoarchitectonic sections to determine areal borders for analysis and the intensity of labeling graded for each region analyzed. Higher magnification images were acquired using an Olympus BX-51 microscope (Olympus, Center Valley, PA) with a Q-Retiga 2000R camera (QImaging, Surrey, BC). Cortical areal boundaries were determined from Nissl stained sections according to the classification of Franklin and Paxinos (2008). Figures were composed in CorelDraw (Corel Corporation, Ottawa, Ontario).

\section{Results}

We examined the distribution of labeling for the Group II metabotropic glutamate receptor subtype, mGluR2, using immunohistochemical methods to identify regions of the developing mouse forebrain that expressed this receptor [4]. Following immunohistochemical staining, we surveyed expression in the developing mouse brain using a Nanozoomer-XR slide scanner (Figure 1). We determined the location of labeled regions in the brain using adjacent Nissl stained sections, which were compared with a standard atlas of the mouse brain [23].

The pattern of mGluR2 labeling in the cerebral cortex exhibited dorsoventral and rostrocaudal differences in the intensity of expression (Figures 1(A)-(C)). In caudal regions, labeling in dorsal cortical areas was weaker than compared with rostral areas, e.g. Figure 1(A)ii versus Figure 1(C)iii. However, the opposite pattern was found when comparing the ventral cortical regions. Intense expression was found in the caudal ventral cortical regions, but was much weaker and nearly absent in the rostral ventral cortical areas, e.g. Figure 1(A)iii versus, Figure 1(C)iii).

The distinction between rostral and caudal patterns of mGluR2 labeling is observed most clearly by comparing sections from rostral and caudal extremes (Figure 2). In the caudal region, ventral cortical labeling is strongly concentrated in entorhinal and adjacent areas (Figure 2(A)). Among the entorhinal cortical areas, the dorsolateral entorhinal (DLEnt), ventral intermediate entorhinal (VIEnt), and medial entorhinal (MEnt) cortical regions all exhibited intense expression for the receptor, while the caudomedial entorhinal cortical regions was very weakly labeled (Figure 2(A)). In addition, the ectorhinal and perirhinal cortical areas were strongly labeled in the caudal cortical regions (Figure 2(A)). In contrast, the rostral sections exhibited very weak labeling of the ventral cortical regions, particularly the piriform cortex (Pir) and the agranular insular cortex (AIP) (Figure 2(B)). In comparison, the dorsal cortical areas were more strongly labeled, particularly in the middle cortical layers (Figure 2(B)). Strong labeling for mGluR2 was present in the somatosensory cortical areas, S1 and S2, and also in the adjacent granular insular cortex, GI (Figure 2(B)).

Further analysis of the labeling in the caudal ventral cortical regions indicates that the expression of mGluR2 in these regions is primarily localized to the lower cortical layers (Figure 3). The ectorhinal cortical area (Ect) exhibits intensely labeled cell bodies in the lower cortical layers, but also has a few labeled cell bodies in upper cortical layers, with labeled fibers extending to the white matter (Figure 3(A)). The weaker labeling in the upper cortical layers continues into the perirhinal cortical area (Prh), but with much weaker fiber labeling (Figure 3(B)). In the dorsolateral entorhinal cortex (DLEnt), labeled cell bodies are intensely packed in the lower half of the cortex, with virtually no labeled cell bodies in the upper half of the DLEnt (Figure 3(C)). This absence of labeling in the upper cortical regions continues into the ventral intermediate (VIEnt) and medial (MEnt) entorhinal cortical areas, however labeling is nearly absent in the caudomedial entorhinal cortical area (CEnt) (Figure 3(B)).

The laminar distribution of mGluR2 labeling in the entorhinal, perirhinal, and ectorhinal cortical areas, contrasts somewhat with that found in the dorsal sensory cortical areas (Figures 2(B), Figure 4). In all the sensory cortical areas examined, mGluR2 labeling was primarily concentrated in layer 4, with weaker labeling in the layer 5 (Figure 4(A) and Figure 4(B)). In these cortical areas, layers 1 - 3 and 6 have weak or no labeling (Figure 4(A) and Figure 4(B)). Within layer 5, lower layer 5B was more heavily labeled than upper layer 5A (Figure 4(A) and Figure 4(B)).

Besides the cortical areas, other regions in the developing mouse brain showed noticeable expression of mGluR2. Many of these labeled structures were limbic-related (Figures 5(A)-(C), Figure 5(E) and Figure 5(F)). For instance, the retrosplenial gyrus (RSG) exhibited intense expression of mGluR2 in fibers extending from the white matter to the cortex (Figure 5(A)), while the mammillary nuclei (MN) (Figure 5(B)) and the basolateral 


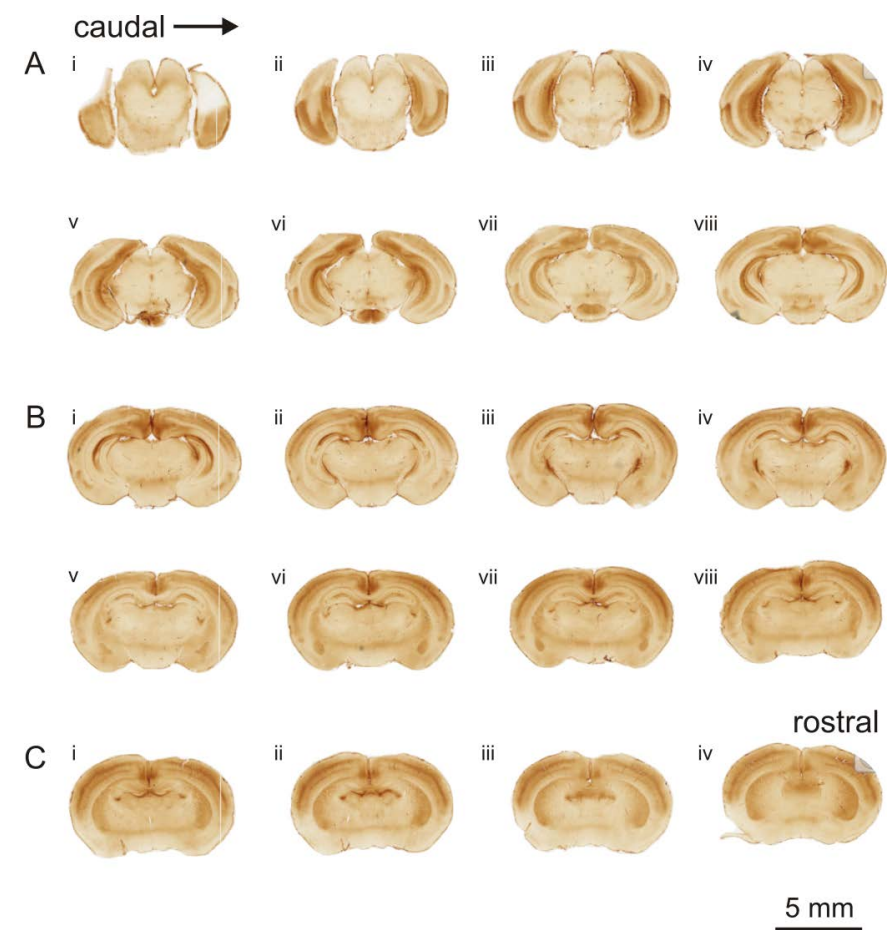

Figure 1. Expression of mGluR2 in the cortex of the developing mouse brain. Slides were scanned on a Nanozoomer-XR slide scanner. Each panel (A)-(C) depicts images scanned from one slide, with the sections mounted caudorostrally from left to right (Ai-viii, Bi-viii, Ci-iv). (A) In caudal sections, intense labeling for mGluR2 is found in the entorhinal cortical areas located in the ventral regions, as well as limbic structures. Labeling of the sensory cortical areas is evident in the dorsal cortical regions; (B) The pattern of labeling in the sensory cortical areas continues in sections containing the thalamus. Strong labeling is also seen in the thalamic reticular nucleus and moderate labeling in the basolateral amygdala; (C) In the rostral most sections, sensory cortical areas are still strongly labeled, but ventral cortical areas are very weakly labeled, particularly the piriform cortex. Additional labeling is found in the caudate putamen, hippocampal commissure, and retrosplenial gyrus.
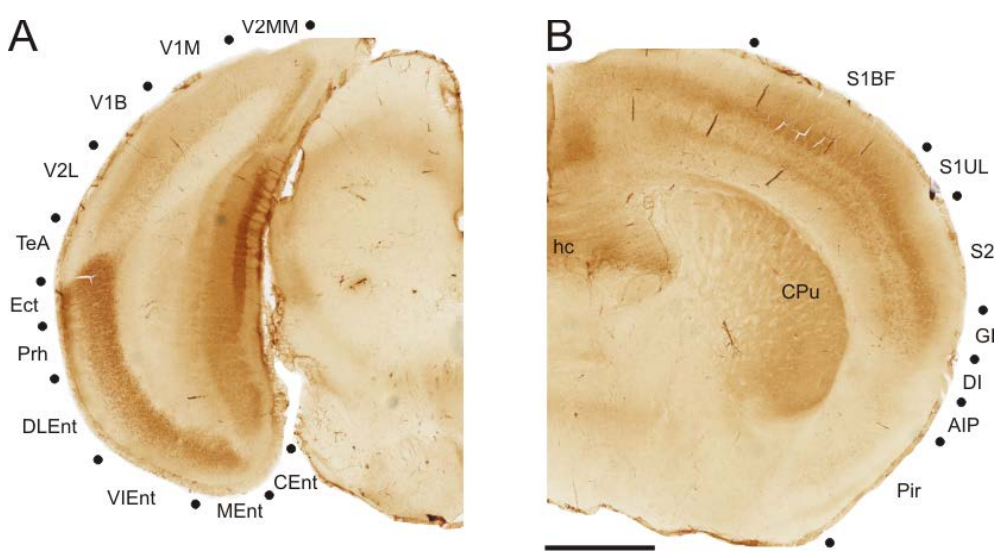

Figure 2. Comparison of mGluR2 labeling from caudal and rostral cortical sections. (A) In the caudal sections, mGluR2 labeling was found most heavily expressed in ventral cortical regions, particularly the entorhinal cortical areas. Section depicts the left hemisphere of that shown in Figure 1(Aiii); (B) In comparison, in rostral sections, mGluR2 labeling is nearly absent from ventral cortical regions, such as the piriform cortex, while the dorsal cortical regions are strongly labeled. Figure depicts the right hemisphere of that shown in Figure 1(Ciii). Abbreviations: AIP, agranular insular cortex; CEnt, caudomedial entorhinal cortex; $C P$, caudate putamen; DI, dysgranular insular cortex; DLEnt, dorsolateral entorhinal cortex; Ect, ectorhinal cortex; GI, granular insular cortex; $h c$, hippocampal commissure; MEnt, medial entorhinal cortex; Pir, pririform cortex; $\mathrm{PrH}$, perirhinal cortex; $S 1 B F$, primary somatosensory cortex barrel field; $S 1 U L$, primary somatosensory cortex upper limb; S2, secondary somatosensory cortex; TeA, temporal association cortex; VIEnt, ventral intermediate entorhinal cortex; $V 1 M$, primary visual cortex monocular area; $V 1 B$, primary visual cortex binocular area; $V 2 L$, secondary visual cortex mediolateral area; $V 2 M M$, secondary visual cortex mediomedial area. 

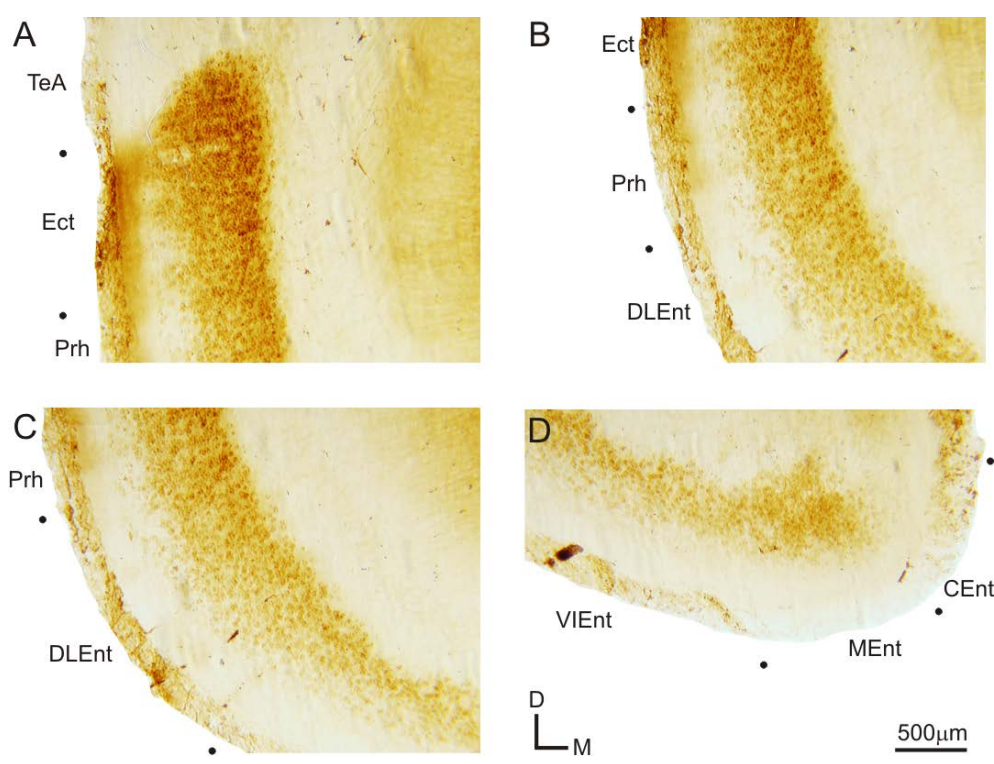

Figure 3. Expression of mGluR2 in caudoventral cortical areas. Higher magnification images of the ventral cortical areas from the section depicted in Figures 1(Aiii) and Figure 3(A). (A) Labeling in the temporal association cortex (TeA), ectorhinal cortex (Ect), and perirhinal cortex (Prh); (B) Expression of mGluR2 in Ect, Prh, and dorsolateral entorhinal cortex (DLEnt); (C) Prh and DLEnt labeling of mGluR2; (D) Ventral intermediate entorhinal cortex (VIEnt), medial entorhinal cortex (MEnt), and caudomedial entorhinal cortex (CEnt) labeling pattern.
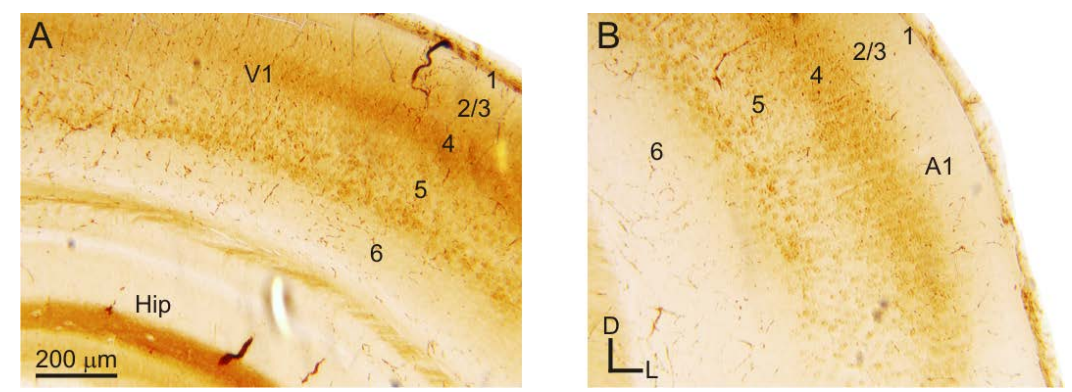

Figure 4. Expression of mGluR2 in sensory cortical areas. (A) Labeling in the primary visual cortex (V1); (B) Labeling in the primary auditory cortex (A1). Panels are higher magnification images from Figure 1(Biii). Additional abbreviations: Hip, hippocampus; 1 - 6, cortical layers 1 - 6 .

amygdala (BLA) (Figure 5(F)) exhibited expression of mGluR2 on cell bodies. Labeled fibers were also found in the presubiculum (PrS) (Figure 5(C)) and the hippocampal commissure (hc) (Figure 5(E)). In addition, the caudate putamen (Figure 3(B)) and the thalamic reticular nucleus (TRN) (Figure 5(D)) were also distinctly labeled for mGluR2.

\section{Discussion}

In this study, we found a unique distribution of mGluR2 in the developing cortex of the mouse. Our findings demonstrated that ventral cortical areas were heavily labeled in the caudal regions of the cortex, while dorsal cortical areas were more heavily labeled in the rostral regions of the cortex. In addition, we found that this mGluR2 labeling was not uniform across the cortex, but varied on a laminar basis, with the upper cortical layers generally devoid of labeling. The labeling was generally found on cell bodies in the cortex, but some labeled fibers were also observed, such as in the retrosplenial gyrus and ectorhinal cortex. Finally, we also observed labeling in several other regions of the brain, particularly limbic-related structures, but also in regions such as the thalamic reticular nucleus and the caudate putamen.

As noted above, in most of the cortical regions surveyed, mGluR2 labeling was found on neuronal cell bodies. These findings match our previous physiological and anatomical studies, which demonstrated a pronounced 

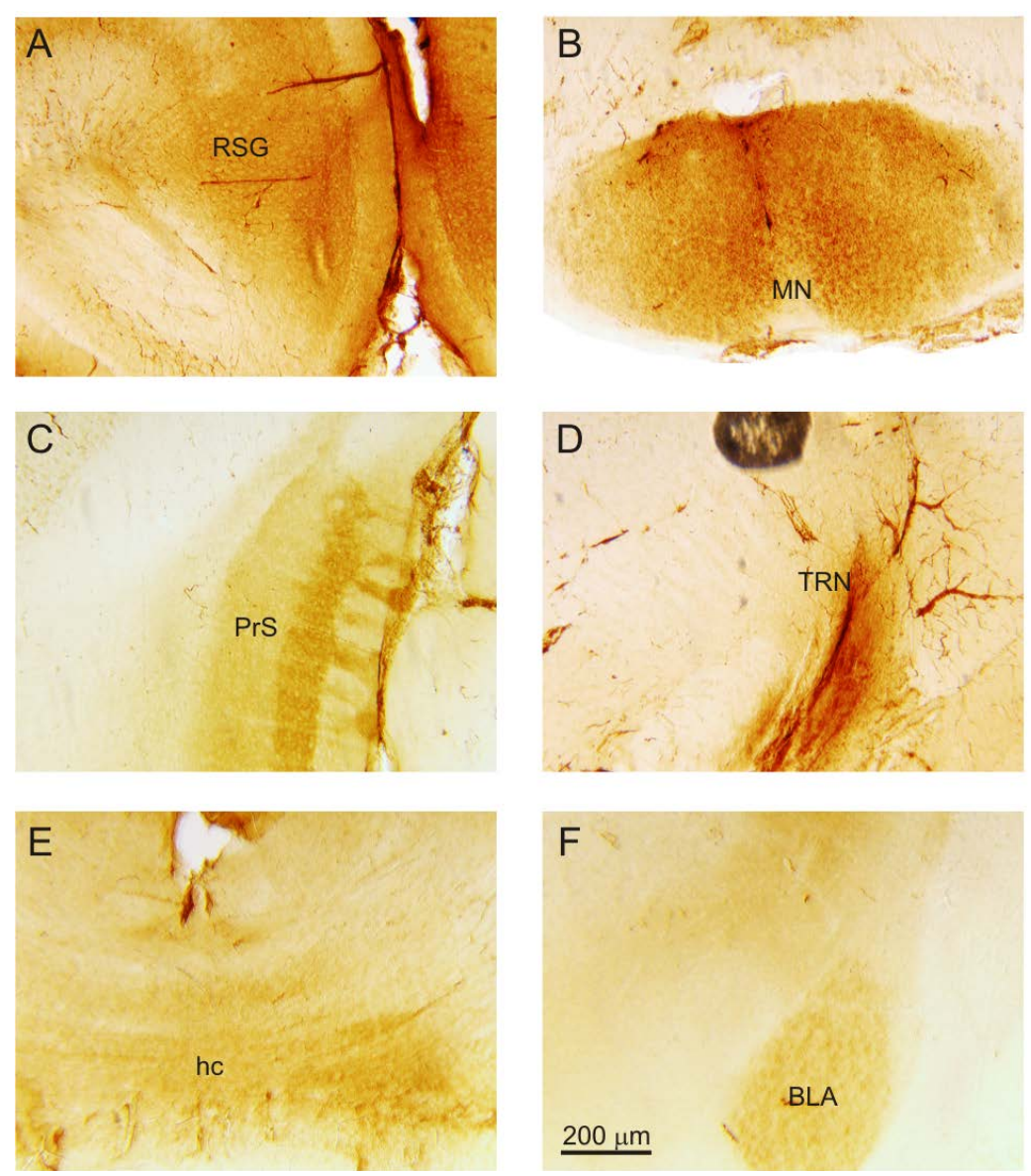

Figure 5. Expression of mGluR2 in other regions of the mouse brain. (A) Labeling in the retrosplenial gyrus (RSG), higher magnification image from Figure 1(Biii); (B) Labeling in the mammillary nuclei $(M N)$, higher magnification image from Figure 1(Avi); (C) Labeling in the presubiculum (PrS), higher magnification image from Figure 1(Aiii); (D) Labeling in the thalamic reticular nucleus (TRN), higher magnification image from Figure 1(Biii); (E) Labeling in the hippocampal commissure $(h c)$, higher magnification image from Figure 1(Ciii); (F) Labeling in the basolateral amygdala (BLA), higher magnification image from Figure 1(Bvii).

postsynaptic localization of mGluR2 in layer 4 of the primary sensory cortical areas [8] [14]. Our current results suggest that mGluR2 is also localized postsynaptically in other sensory cortical layers, the entorhinal cortical areas, the mammillary bodies, and the amygdala, among other sites. In general, Group II mGluRs are localized pre-synaptically in other regions of the brain [2] [4] [9]-[11]. The pre-synaptic activation of Group II mGluRs reduces the probability of synaptic release through autoreceptor activation during periods of high activity [14] [18]. Postsynaptic Group II mGluRs may serve an analogous function, but instead could enable gain control at multiple synaptic sites through the hyperpolarization of the postsynaptic neuronal cell membrane [8]. Thus, the localization of mGluR2 to cell bodies in regions such as the entorhinal cortex or amygdala may have important functions for enabling gain control at their synapses. Moreover, these receptors have also been implicated in numerous other functions, such as plastic reorganization and developmental modeling of the synapse [2] [7] [18] [20]-[22] [24]-[26], and thus may serve similar functions in those regions identified in this study.

The observed localization of these receptors to limbic-related structures suggests that these receptors are particularly important for information processing in these regions. In particular, these receptors have been implicated as important in various neurological disorders, such as schizophrenia, and their appearance in these regions may hint at their crucial role for the proper functioning of these circuits [7] [19] [27]. Alternatively, their combined expression in these regions in the developing brain may act as a molecular signal for the development of these circuits in the mature brain [28] [29]. Finally, we observed that these receptors were present in the sensory cortical areas of the mouse. These findings are similar to those that we have reported previously [8]. The 
localization of these receptors to those regions may be crucial to information processing at these synapses and perhaps distinguish information-bearing from non-information bearing pathways [30] [31].

\section{Conclusion}

In this study, we found a differential distribution of the Group II metabotropic glutamate receptor, mGluR2, in the developing cerebral cortex of the mouse. This receptor was found highly expressed in ventral caudal cortical regions and dorsal rostral cortical regions, as well as other structures in the brain, particularly those associated with limbic functions. With the neuroanatomical localization of the receptors to these structures, future studies should now investigate the physiological, behavioral and developmental significance of activating or inactivating these receptors in these regions of the brain.

\section{Acknowledgements}

We thank X. Wu and S. Ring for their assistance with the departmental core histology and microscopy centers. We thank the support of the Supervised Undergraduate Research Experience (SURE) program grant for Ms. Venkatadri from the Louisiana Board of Regents (LEQSF-EPS(2013)-SURE-77). Additional support for this work was provided by NIH/NIDCD grant R03 DC 11361, Louisiana Board of Regents RCS grant RD-A-09, SVM CORP-USDA 1433 grant LAV 3493, and Action on Hearing Loss Grant RNID F42.

\section{References}

[1] Nakanishi, S. (2004) Molecular Diversity of Glutamate Receptors and Implications for Brain Function. Science, 258, 597-693. http://dx.doi.org/10.1126/science.1329206

[2] Cartmell, J. and Schoepp, D.D. (2000) Regulation of Neurotransmitter Release by Metabotropic Glutamate Receptors. Journal of Neurochemistry, 75, 889-907. http://dx.doi.org/10.1046/j.1471-4159.2000.0750889.X

[3] Conn, P.J. and Pin, J.P. (1997) Pharmacology and Functions of Metabotropic Glutamate Receptors. Annual Review of Pharmacology and Toxicology, 37, 205-237. http://dx.doi.org/10.1146/annurev.pharmtox.37.1.205

[4] Neki, A., Ohishi, H., Kaneko, T., Shigemoto, R., Nakanishi, S. and Mizuno, N. (1996) Pre- and Postsynaptic Localization of a Metabotropic Glutamate Receptor, mGluR2, in the Rat Brain: An Immunohistochemical Study with a Monoclonal Antibody. Neuroscience Letters, 202, 197-200. http://dx.doi.org/10.1016/0304-3940(95)12248-6

[5] Ngomba, R.T., Santolini, I., Salt, T.E., Ferraguti, F., Battaglia, G., Nicoletti, F. and van Luijtelaar, G. (2011) Metabotropic Glutamate Receptors in the Thalamocortical Network: Strategic Targets for the Treatment of Absence Epilepsy. Epilepsia, 52, 1211-1222. http://dx.doi.org/10.1111/j.1528-1167.2011.03082.x

[6] Lee, C.C. and Sherman, S.M. (2009) Modulator Property of the Intrinsic Cortical Projections from Layer 6 to Layer 4. Frontiers in Systems Neuroscience, 3, 3. http://dx.doi.org/10.3389/neuro.06.003.2009

[7] Sherman, S.M. (2014) The Function of Metabotropic Glutamate Receptors in Thalamus and Cortex. Neuroscientist, 20, 136-149. http://dx.doi.org/10.1177/1073858413478490

[8] Lee, C.C. and Sherman, S.M. (2009) Glutamatergic Inhibition in Sensory Neocortex. Cerebral Cortex, 19, $2281-2289$. http://dx.doi.org/10.1093/cercor/bhn246

[9] Petralia, R.S., Wanga, Y.-X., Niedzielskia, A.S. and Wentholda, R.J. (1996) The Metabotropic Glutamate Receptors, MGLUR2 and MGLUR3, Show Unique Postsynaptic, Presynaptic and Glial Localizations. Neuroscience, 71, 949-976. http://dx.doi.org/10.1016/0306-4522(95)00533-1

[10] Ryo, Y., Miyawaki, A., Furuichi, T. and Mikoshiba, K. (1993) Immunohistochemical Localization of Metabotropic and Ionotropic Glutamate Receptors in the Mouse Brain. Annals of the New York Academy of Sciences, 707, 554-556. http://dx.doi.org/10.1111/j.1749-6632.1993.tb38124.x

[11] Shigemoto, R., Kinoshita, A., Wada, E., Nomura, S., Ohishi, H., Takada, M., Flor, P.J., Neki, A., Abe, T., Nakanishi, S. and Mizuno, N. (1997) Differential Presynaptic Localization of Metabotropic Glutamate Receptor Subtypes in the Rat Hippocampus. Journal of Neuroscience, 17, 7503-7522.

[12] Dutar, P., Petrozzino, J.J., Vu, H.M., Schmidt, M.F. and Perkel, D.J. (2000) Slow Synaptic Inhibition Mediated by Metabotropic Glutamate Receptor Activation of GIRK Channels. Journal of Neurophysiology, 84, 2284-2290.

[13] Simonyi, A., Ngomba, R.T., Storto, M., Catania, M.V., Miller, L.A., Youngs, B., DiGiorgi-Gerevini, V., Nicoletti, F. and Sun, G.Y. (2005) Expression of Groups I and II Metabotropic Glutamate Receptors in the Rat Brain during Aging. Brain Research, 1043, 95-106. http://dx.doi.org/10.1016/j.brainres.2005.02.046

[14] Lee, C.C. and Sherman, S.M. (2012) Intrinsic Modulators of Auditory Thalamocortical Transmission. Hearing Re- 
search, 287, 43-50. http://dx.doi.org/10.1016/j.heares.2012.04.001

[15] Beaver, C.J., Ji, Q. and Daw, N.W. (1999) Effect of the Group II Metabotropic Glutamate Agonist, 2R,4R-APDC, Varies with Age, Layer, and Visual Experience in the Visual Cortex. Journal of Neurophysiology, 82, 86-93.

[16] Farazifard, R. and Wu, S.H. (2010) Metabotropic Glutamate Receptors Modulate Glutamatergic and GABAergic Synaptic Transmission in the Central Nucleus of the Inferior Colliculus. Brain Research, 1325, 28-40. http://dx.doi.org/10.1016/j.brainres.2010.02.021

[17] Lu, Y. (2014) Metabotropic Glutamate Receptors in Auditory Processing. Neuroscience, 274, 429-445. http://dx.doi.org/10.1016/j.neuroscience.2014.05.057

[18] Mateo, Z. and Porter, J.T. (2007) Group II Metabotropic Glutamate Receptors Inhibit Glutamate Release at Thalamocortical Synapses in the Developing Somatosensory Cortex. Neuroscience, 146, 1062-1072. http://dx.doi.org/10.1016/j.neuroscience.2007.02.053

[19] Niswender, C.M. and Conn, P.J. (2010) Metabotropic Glutamate Receptors: Physiology, Pharmacology, and Disease. Annual Review of Pharmacology and Toxicology, 50, 295-322. http://dx.doi.org/10.1146/annurev.pharmtox.011008.145533

[20] Otani, S., Daniel, H., Takita, M. and Crépel, F. (2002) Long-Term Depression Induced by Postsynaptic Group II Metabotropic Glutamate Receptors Linked to Phospholipase C and Intracellular Calcium Rises in Rat Prefrontal Cortex. Journal of Neuroscience, 22, 3434-3444.

[21] Renger, J., Hartman, K.N., Tsuchimoto, Y., Yokoi, M., Nakanishi, S. and Hensch, T.K. (2002) Experience-Dependent Plasticity without Long Term Depression by Type 2 Metabotropic Glutamate Receptors in Developing Visual Cortex. Proceedings of the National Academy of Sciences of the United States of America, 99, 1041-1046. http://dx.doi.org/10.1073/pnas.022618799

[22] Sekizawa, S., Bechtold, A.G., Tham, R.C. and Bonham, A.C. (2009) A Novel Postsynaptic Group II Metabotropic Glutamate Receptor Role in Modulating Baroreceptor Signal Transmission. Journal of Neuroscience, 29, 11807-11816. http://dx.doi.org/10.1523/JNEUROSCI.2617-09.2009

[23] Franklin, K.B.J. and Paxinos, G. (2001) The Mouse Brain in Stereotaxic Coordinates. 2nd Edition, Academic Press, New York.

[24] Bandrowski, A.E., Aramakis, V.B., Moore, S.L. and Ashe, J.H. (2001) Metabotropic Glutamate Receptors Modify Ionotropic Glutamate Responses in Neocortical Pyramidal Cells and Interneurons. Experimental Brain Research, 136, 25-40. http://dx.doi.org/10.1007/s002210000556

[25] Frank, E.T., Newell, K.A. and Huang, X.F. (2011) Density of Metabotropic Glutamate Receptors 2 and 3 (mGluR2/3) in the Dorsolateral Prefrontal Cortex Does Not Differ with Schizophrenia Diagnosis but Decreases with Age. Schizophrenia Research, 128, 56-60. http://dx.doi.org/10.1016/j.schres.2011.01.008

[26] Nishimaki, T., Jang, I.S., Ishibashi, H., Yamaguchi, J. and Nabekura, J. (2007) Reduction of Metabotropic Glutamate Receptor-Mediated Heterosynaptic Inhibition of Developing MNTB-LSO Inhibitory Synapses. European Journal of Neuroscience, 26, 323-330. http://dx.doi.org/10.1111/j.1460-9568.2007.05656.x

[27] Matosin, N., Fernandez-Enright, F., Frank, E., Deng, C., Wong, J., Huang, X.F. and Newell, K.A. (2014) Metabotropic Glutamate Receptor mGluR2/3 and mGluR5 Binding in the Anterior Cingulate Cortex in Psychotic and Nonpsychotic Depression, Bipolar Disorder and Schizophrenia: Implications for Novel mGluR-Based Therapeutics. Journal of Psychiatry \& Neuroscience, 39, 407-416. http://dx.doi.org/10.1503/jpn.130242

[28] Koyama, R., Yamada, M.K., Nishiyama, N., Matsuki, N. and Ikegaya, Y. (2002) Group II Metabotropic Glutamate Receptor Activation Is Required for Normal Hippocampal Mossy Fibre Development in the Rat. Journal of Physiology, 539, 157-162. http://dx.doi.org/10.1113/jphysiol.2001.013505

[29] Reid, S.N. and Romano, C. (2001) Developmental and Sensory-Dependent Changes of Group II Metabotropic Glutamate Receptors. Journal of Comparative Neurology, 429, 270-276. http://dx.doi.org/10.1002/1096-9861(20000108)429:2<270::AID-CNE7>3.0.CO;2-W

[30] Lee, C.C. and Sherman, S.M. (2010) Drivers and Modulators in the Central Auditory Pathways. Frontiers in Neuroscience, 4, 79-86. http://dx.doi.org/10.3389/neuro.01.014.2010

[31] Lee, C.C. and Sherman, S.M. (2011) On the Classification of Pathways in the Auditory Midbrain, Thalamus, and Cortex. Hearing Research, 276, 79-87. http://dx.doi.org/10.1016/j.heares.2010.12.012 
Scientific Research Publishing (SCIRP) is one of the largest Open Access journal publishers. It is currently publishing more than 200 open access, online, peer-reviewed journals covering a wide range of academic disciplines. SCIRP serves the worldwide academic communities and contributes to the progress and application of science with its publication.

Other selected journals from SCIRP are listed as below. Submit your manuscript to us via either submit@scirp.org or Online Submission Portal.
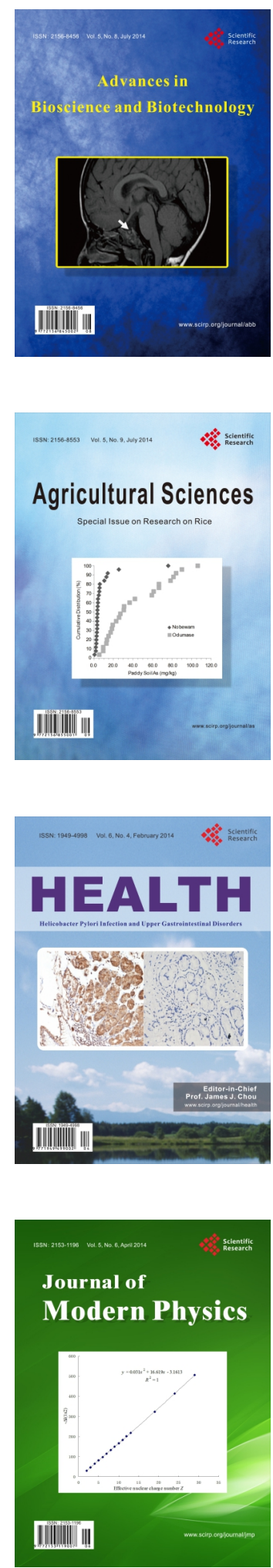
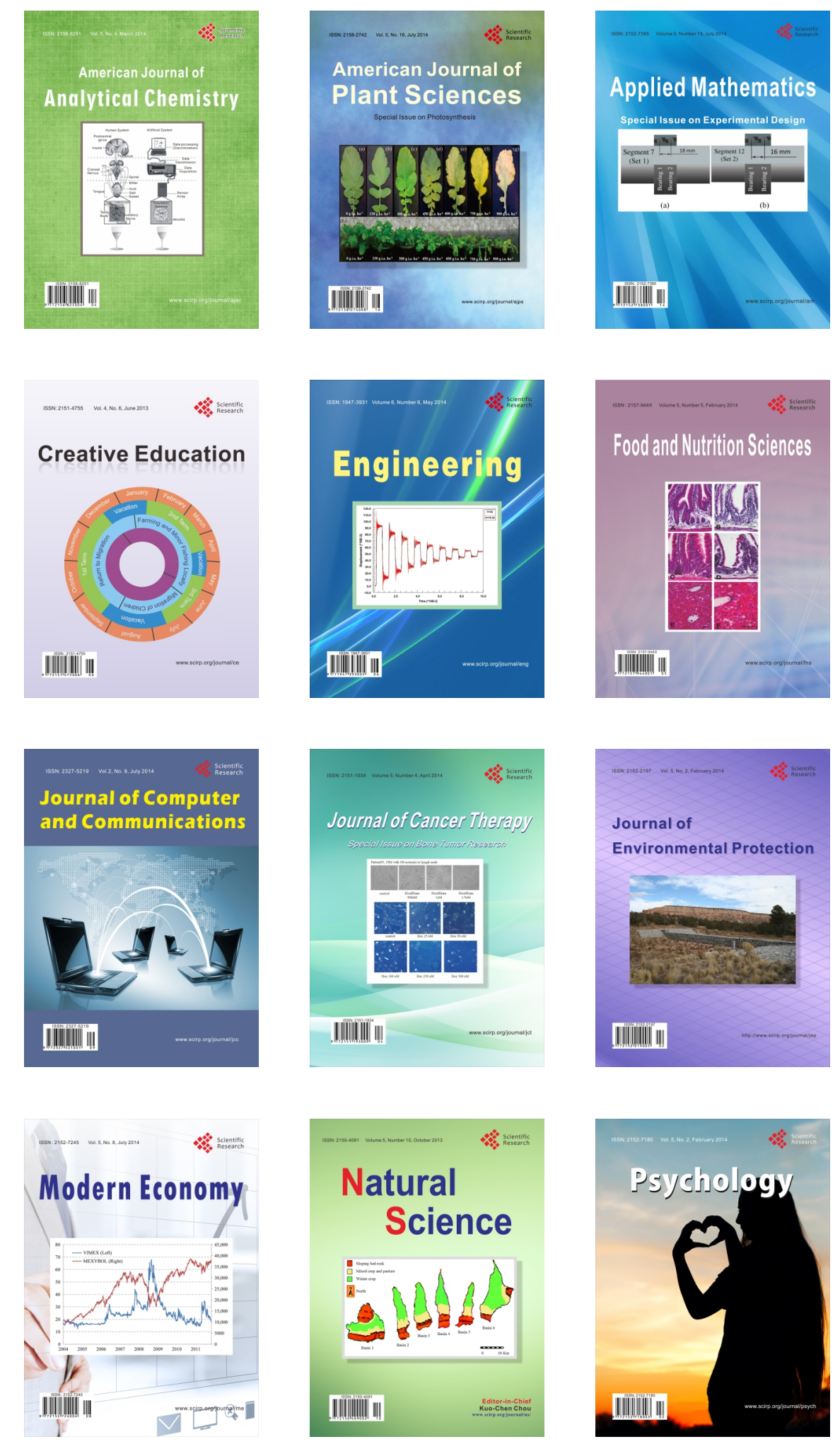